\title{
AN INSIGHT INTO THE KINSHIPS OF THE FUNGI
}

Riddhi Datta*, Joy Sarkar" and Krishnendu Acharya*

Abstract | The evolutionary origin of fungi is important in determining the phylogenetic relationships between fungi, animals and plants. However, determining the true relationship of fungi has been somewhat difficult owing to their simple morphology and presence of convergent characters. With the advent of newer molecular techniques, analysis of conserved protein sequences, cytochrome systems, mitochondrial and nuclear genetic material and rRNAs are being employed in elucidating phylogenetic kinships among the eukaryotes. These emerging evidences suggest that the derivation of the fungi from plants or algae would require more evolutionary changes than its derivation from protozoa, the unicellular ancestor of the animals. To gain an overview of the current state of the science, we have extensively reviewed the existing literatures and it appeared to us that fungi are more closely related to animals than to plants.

*Molecular and Applied Mycology and Plant Pathology Laboratory, Department of Botany, University of Calcutta, Kolkata-700019.

Correspondence to K.A. e-mail:

krish_paper@yahoo.com
In the year of 1588 , Giambattista della Porta, an Italian versatile scientist, suggested in his book Phytognomonica that, "fungi, since they do not arise from seed, are children of the God"1. Although for $21^{\text {st }}$ century, the statement possesses less scientific value but the underlying facts of the statement remain somewhat unchanged even today. Even today the origin \& evolutionary relationships of fungi remains a matter of speculation and problem of future research for mycologists.

The evolutionary origin of fungi is important in determining the phylogenetic relationships between fungi, animals and plants. It has generally been observed that both in cases of plants and animals evolution have led to the generation of complex structures from the simpler ones. This view of progressive evolution prompts us to look for simple primitive organisms which might have been the probable 
ancestor or the basal group from which other organisms have evolved subsequently. At the same time retrogression from complex to simpler structures might also have taken place in the course of evolution.

Initially it was believed that photosynthetic organisms were the first to evolve on earth since all other heterotrophs depend on them for food. This view of 'Algal Origin' of fungi is mainly based on their morphological similarity and simplicity and assumes that the fungi evolved from algae by gradual loss of chlorophyll ${ }^{2}$. According

On the basis of cell wall component

The most important components of fungal cell wall are polysaccharides, among which chitin is the predominant one. The fungal chitin is composed of an unbranched polymer of $\beta-1,4$ linked $N$ acetylglucosamine units, which is distinct from higher plants. The chitin present in fungal cell wall is primarily of the $\alpha$-form while the $\beta$-form is generally absent. The $\alpha$ chitin is also abundantly present in animals ${ }^{15}$. to this view, the Chytridiales have evolved from unicellular algae, the Saprolegniales from the Siphonales, the Zygomycetes from the Conjugales and the higher fungi from the red algae ${ }^{3-}$ ${ }^{6}$. The second school of thought suggests the derivation of fungi from non-chlorophyllous unicellular forms ${ }^{7-8}$. It was Gobi (1884) who first proposed that fungi were derived independently from the Protozoa ${ }^{9}$. This view was subsequently supported by many workers $^{10-13}$. The key evidences in support of a fungi-animalia clade includes analysis of protein sequences of

Among animals, chitin is the principle component of the hard exoskeletons of nearly a million species of arthropods, both insects and arachnids, and is probably the second most abundant polysaccharide, next only to cellulose. The most striking similarity between the plant and fungal cell walls is the presence of cellulose. However, fungal cellulose is somewhat different from that found in plant cell walls. In fact, fungal cellulose is less biosynthetic pathways, cytochrome systems, mitochondrial and nuclear genetic material, rRNA, biochemical and structural cellular features, glycoproteins, mode of nutrition and storage of nutritive materials. These existing evidences suggests that the derivation of the fungi from plants or algae would require more evolutionary changes than its derivation from protozoa, the unicellular ancestor of the animals ${ }^{14}$. There are striking evidences in support of this fungi-animalia clade based primarily on the biochemical analyses.

crystalline than plant cellulose, as evident from $\mathrm{X}$-ray diffraction images ${ }^{15}$.

\section{On the basis of polysaccharide microfibrils}

It is well known that the polysaccharide chains of cell wall are grouped together to form micelles. These micelles are again grouped to form microfibrils which together form a network while the matrix is formed by pectin-like substances. In fungi, the 
polysaccharide

microfibrils are randomly oriented. In contrast, plants and algae possess microfibrils that are laid down in parallel arrays ${ }^{15}$. When fungal polysaccharides were treated with alkalis containing nitrogen, degradation occurred along with the production of acetic acid. The results differ when same reaction is carried out with plant polysaccharides ${ }^{15}$.

Two types of bonding patterns are evident in the fungal glycoproteins: oligosaccharides are joined to serine or threonine residues through an O-glycosidic linkage and sugar present at the reducing end of the oligosaccharide moiety is joined to an asparagines residue through an $\mathrm{N}$ linkage ${ }^{15}$. This pattern is similar to those of glycoproteins of animal origin.

\section{On the basis of mode of nutrition}

The process of photosynthesis is very common and important in plant life as it serves as their source for food. Just like animals, fungi lack chloroplasts. Thus, the photosynthesizing machinery is absent in them. Therefore, their mode of nutrition involves the following sequence: photosynthesis in plants, ingestion in animals and absorption in fungi.

\section{On the basis of reserve} food

Many eukaryotic organisms, including fungi, accumulate lipids as carbon reserve ${ }^{16}$. One of the major storage products of fungi, bacteria, protozoa and higher animals is glycogen. On the other hand, plants predominantly store starch $^{15}$. Both glycogen and starch are polymers of glucose residues. However, the main structural difference between these two storage compounds is that, glycogen is more compact and more extensively branched (branches occur every 8 to 12 residues) in comparison to starch. Also, starch is composed of two types of glucose polymers: amylose and amylopectin and glycogen are just like amylopectin.

On the basis of mitochondrial cristae and plastids

Fungi share many common morphological and structural cellular features with animals. Non-discoidal plate-like mitochondrial cristae are present in both fungi and animals, while plants have mostly tubular mitochondrial cristae. This finding explains the origin of other crystal types as the consequence of co-evolution of the plastid and mitochondrial trans-envelope protein systems.

In both fungi and animals, plastids are absent, while plants possess plastids with two envelopes, usually containing starch $^{17}$. This also supports the hypothesis of a closer resemblance between fungi and animals.

\section{On the basis of cytochrome system}

In a study made by Boulter and Derbyshire (1957), the visible absorption of cytochromes of fungi was compared to the spectra obtained from yeasts and animals $^{18}$. This intensive spectroscopic analysis revealed that 45 fungal species studied, representing all major classes, possessed a cytochrome structure similar to that of mammalian and avian 
cells. In contrast, the structure differed from the structure of the plant cytochromes (FIG.1).

The b-type and c-type cytochromes present in fungi are also found in metazoan but is apparently absent in plants. Thus, it can be concluded that these features may be a biochemical parameter for phylogenetic affinities between fungi and animals.

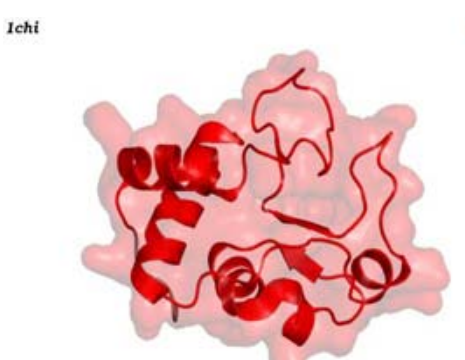

A

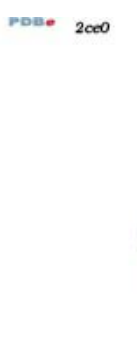

tchi pose.

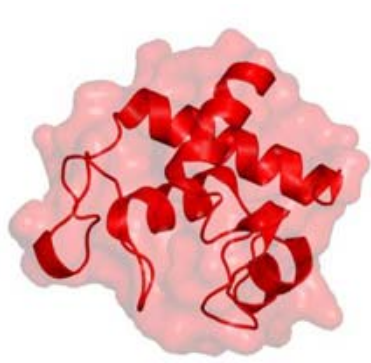

B
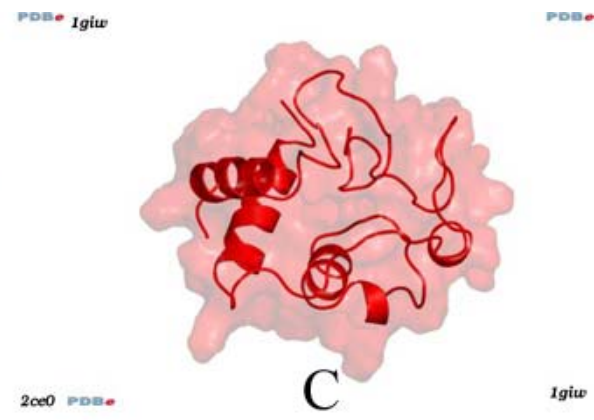

Figure 1 | Structure of cytochrome C (monodomain): (A) Saccharomyces cerevisiae; (B) Arabidopsis thaliana; (C) Equus caballus (http//:pdbe.org).

\section{On the basis of origin of sterols}

Like mammals, fungal sterols are generally derived from lanostane. In fact, their biosynthetic pathways are also somewhat similar, where sterols arise from lanosterol by the loss of their methyl groups, two on the C-14 along with other structural alterations ${ }^{19}$. In contrast, in plants and algae, sterols are biosynthetically derived from a cycloartane derivative, cycloartenol, involving loss of the equivalent three methyl groups and the opening of the $9 \beta, 19$-cyclopropane $\operatorname{ring}^{19}$.

A recent study has shown that sterols are synthesized from the common precursor squalene. A key step in the biosynthetic pathway involves a P450 enzyme (CYP51) which uses lanosterol in Animals, Fungi, and Stramenopiles, and obtusifoliol in green plants as substrates ${ }^{20}$.

$\begin{aligned} & \text { On the basis of } \\ & \text { biosynthesis }\end{aligned}$
$\begin{aligned} & \text { of } \\ & \text { polyunsaturated fatty } \\ & \text { acids }\end{aligned}$

Fungi show considerable similarities to animals in the biosynthesis of polyunsaturated fatty acids. Two distinct pathways are recognized in this respect: one for the production of $\alpha$-linolenic acid, and the other for the $\gamma$-linolenic acid and arachidonic acid (FIG. 2).
Lejohn (1974) observed that of these two forms, the $\alpha$-linolenic acid is predominantly synthesized by higher plants but is absent in protozoa and metazoa. On the contrary, $\gamma$ linolenic acid appears to be abundantly synthesized by animals ${ }^{21}$. Shaw (1965) and Lejohn (1974) have independently shown that out of 31 species of fungi (7 orders of the Ascomycota, 5 orders of the Basidiomycota and others), all produce $\gamma$ linolenic acid, and not the $\alpha$-linolenic acid ${ }^{21-22}$. 


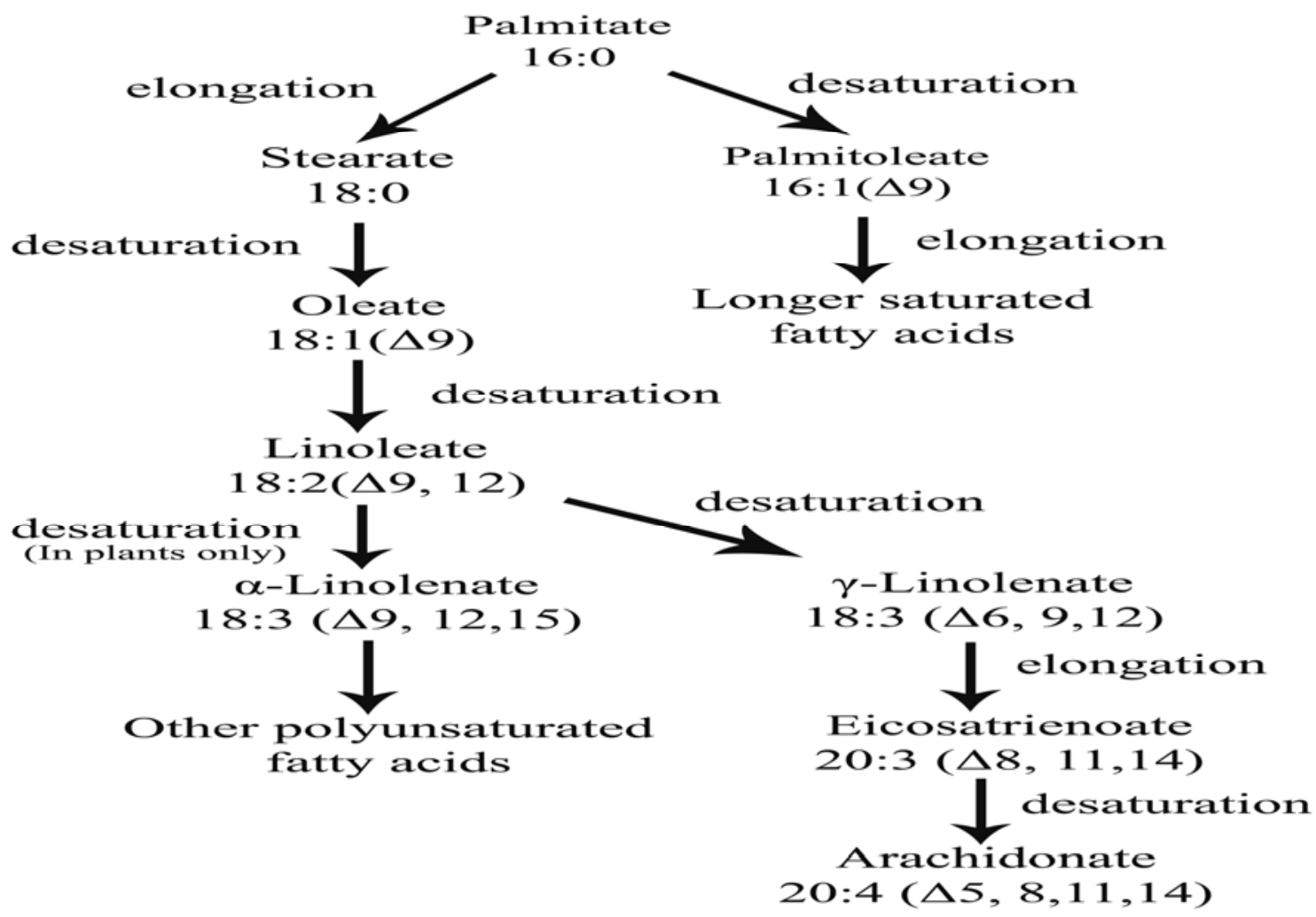

Figure 2 | Routes of synthesis of polyunsaturated fatty acids. Palmitate is the precursor of stearate and longer-chain saturated fatty acids, as well as the monounsaturated acids, palmitoleate and oleate. Mammals \& fungi cannot convert oleate into linolenate, which are therefore required in the diet as essential fatty acids. Conversion of linoleate into other polyunsaturated fatty acids and eicosanoids is outlined here.

\section{On the basis of biosynthesis of L-lysine}

The distribution of two distinct pathways leading to the biosynthesis of Llysine has also been cited as evidence in favour of fungi-animalia clade. The diaminopimelic acid (DAP) route is the most common among both monocotyledonous and dicotyledonous plants. On the other hand, the second, aminoadipic acid (AAA) route, is confined to the fungi ${ }^{23}$. On this basis, it has been suggested that fungi are distinct from plants. Although the complete
AAA route is confined to fungi, elements of the AAA pathway of lysine degradation are found in animals as well ${ }^{21}$. It can be assumed that the AAA lysine pathway has originated as a result of the loss of DAP during the conversion of the peptidoglycan wall into an animal-like chitinous exoskeleton $^{24}$.

\section{On the basis of sequence analysis of proteins}

Baldauf and Palmer studied the phylogenetic relationships between plants, animals and fungi on the basis of the sequences of 25 proteins $^{25}$. They showed that 4 insertions / deletions are uniquely shared by animals and fungi, relative to plants, protests and bacteria. These insertion / deletions were located in the genes encoding 4 conserved proteins namely actin, $\alpha$ tubulin, $\beta$-tubulin and elongation factor $1 \alpha$ and the result place fungi and animals together as a monophyletic group and excludes plants and a broad diversity of protists from them ${ }^{25}$. This view is also supported by the observation that proteins 
like $\quad \beta$-tubulin and calmodulin are all conserved across the fungal and animal kingdom and even in their human counterparts ${ }^{26}$. The phylogenetic tree of diverse eukaryotes based on $\beta$-tubulin using maximum likelihood method also suggests that fungi including the primitive microsporidia are more closely related to animals ${ }^{27}$.

\section{On the basis of heat shock proteins}

The heat shock protein 70 (Hsp 70) are ubiquitous in their presence and the sequences show a high degree of conservation. Sequencing and analysis of the entire nucleic acid sequence of cytoplasmic Hsp $\quad 70$ from 8 diploblastic species supports the monophyly of diploblastic animals as well as a close phylogenetic relationship between animals and fungi ${ }^{28}$. While studying the phylogenetic relationship and the branching order between animals, plants, and fungi, they aligned the sequences of some "lower" metazoan taxa with existing Hsp70 orthologous sequences from a range of higher plants and fungi and several sequences of triploblastic animals that were extracted from GenBank. They constructed phylogenetic trees using bootstrap and parsimony methods and both the methods revealed similar branching orders. The high bootstrap score (91\%) of the branch point leading to the animalsfungi clade indicated that the affinities between these groups are robust and significant. The parsimony tree also supported an animal-fungi clade. However, the support for this was not statistically robust $(80 \%)$. Simpson et al (2006) used maximum likelihood method to construct a phylogenetic tree based on gene sequences of Hsp 70, Hsp 90 and four other slowly evolving genes. This study also suggests a closer relation of fungi with animals than with plants $^{29}$.

\section{On the basis of translation elongation factor 3}

The soluble translation elongation factor 3 (EF-3) appears to be uniquely present in the fungi and exhibits ribosome dependent ATPase and CTPase activities that are not intrinsic to the fungal ribosome but are essential for translation elongation in vivo. This EF-3 has been identified in a wide range of fungal species ${ }^{26}$, ${ }^{30}$. It has been found that fungal EF-3 "displays amino acid similarity to myosin proteins of animals whose cellular function is to provide the motile force of muscle," and which work there mainly with actin ${ }^{26}$. Also, EF-3 "have at least ten regions of detectable amino acid sequence similar to the mammalian myosin heavy-chain protein (MyHC),"26. However, these regions are not responsible for a defined myosin activity and only "occur in equivalent positions in mammalian and yeast myosin proteins". These findings also support the hypothesis that the members of Dictyosteliomycetes, the cellular slime molds, are more closely related to the Animalia-Fungi clade and are distantly related to Plantae ${ }^{31}$.

\section{On the basis of UGA codes}

In 1987, Cavalier-Smith ${ }^{17}$ suggested that fungal mitochondrial codon UGA codes for tryptophan and is not a chain terminator. This dissimilarity with plants 
was also supported by Lang (1984) ${ }^{32}$. According to Scazzacchio (1987), all animal mitochondrial UGA are also read as tryptophan $^{33}$. These reports prompt that fungi are closer to animals than to plants, at least on the basis of UGA codes. However, Inagaki et al (1998) suggested that the ancestral mitochondrion was bearing the universal genetic code and subsequently reassigned the codon to Tryptophan independently, at least in fungi and few other lineages ${ }^{34}$. As such the organisms with the modified UGA code cannot be considered monophyletic.

\section{A Phylogenetic consideration}

The existing controversies over the phylogenetic relationships of fungi encouraged several molecular phylogenics to further investigate the various aspects of evolution and to find more convincing kinships of the fungi.

The early diversification of the eukaryotes is one of the major events in course of evolution. The 'Crownstem' model of eukaryotic phylogenys was proposed in the early 1990s based on the study of small subunit rRNA sequences $^{35-37}$. According to this model, the plants, animals and fungi form a 'crown group' in the eukaryotic tree and that they separated from each other more recently than some early branching protists. According to the 'Crown-stem' model it was estimated that the origin of eukaryotes occurred at approximately 2,700 million years ago and that the plants and animals/fungi separated approximately $\quad 1,600$ million years ago.

A more recent phylogenetic study revealed that the eukaryotes can be classified into 6 supergroups, namely, Archaeplastida which includes plants and green algae, Opisthokonta which includes animals and fungi, and 4 other supergroups: Excavata, Rhizaria, Chromalveolata and Amoebozoa ${ }^{38}$ (Adl et al, 2005). This view is based mainly on the molecular and morphological-cell biological evidences. Further studies suggest that the number of supergroups might be more than 6 and would have diverged during the early phase of eukaryotic evolution that is sometimes described as 'Big Bang' event ${ }^{39}$. In fact, the split between the Archaeplastida and Opisthokonta is among the earliest known eukaryotic divergences, before the divergence of other protist groups from either of them. Thus this model predicts that the separation of plants from animal/fungi would be much more ancient than what was suggested by the 'Crown-stem' model.

Apart from their kinships with other groups of organisms, the evolutionary relationships among different groups of fungi are also somewhat controversial.

Traditionally it was believed that fungi with flagellated cells (Chytridiomycota) are the sister group to the remaining phyla of nonflagellated fungi

(Zygomycota, Glomeromycota,

Ascomycota and Basidiomycota) which indicates that a single loss of flagellum was coincident with a migration to land. However, James et al (2006) estimated the phylogeny of fungi using data from 6 gene regions: 18S rRNA, 28S rRNA, 
5.8S rRNA, EF-1 $\alpha$ and two RNA polymerase II subunits (RPB1 and RPB2 $)^{40}$. The results supported monophyly of Ascomycota, Basidiomycota and Glomeromycota (FIG. 3). The Ascomycota and
Basidiomycota formed a clade 'dikarya'. The analysis also supported a clade uniting 'dikarya' and Glomeromycota as suggested by earlier studies with $18 \mathrm{~S}$ rRNA. It also supported the Opisthokont clade which includes fungi, metazoa and choanoflagellida. It further suggests that Chytridiomycota is not monophyletic consisting of early diverging lineages that have retained the zoospore ${ }^{40}$.

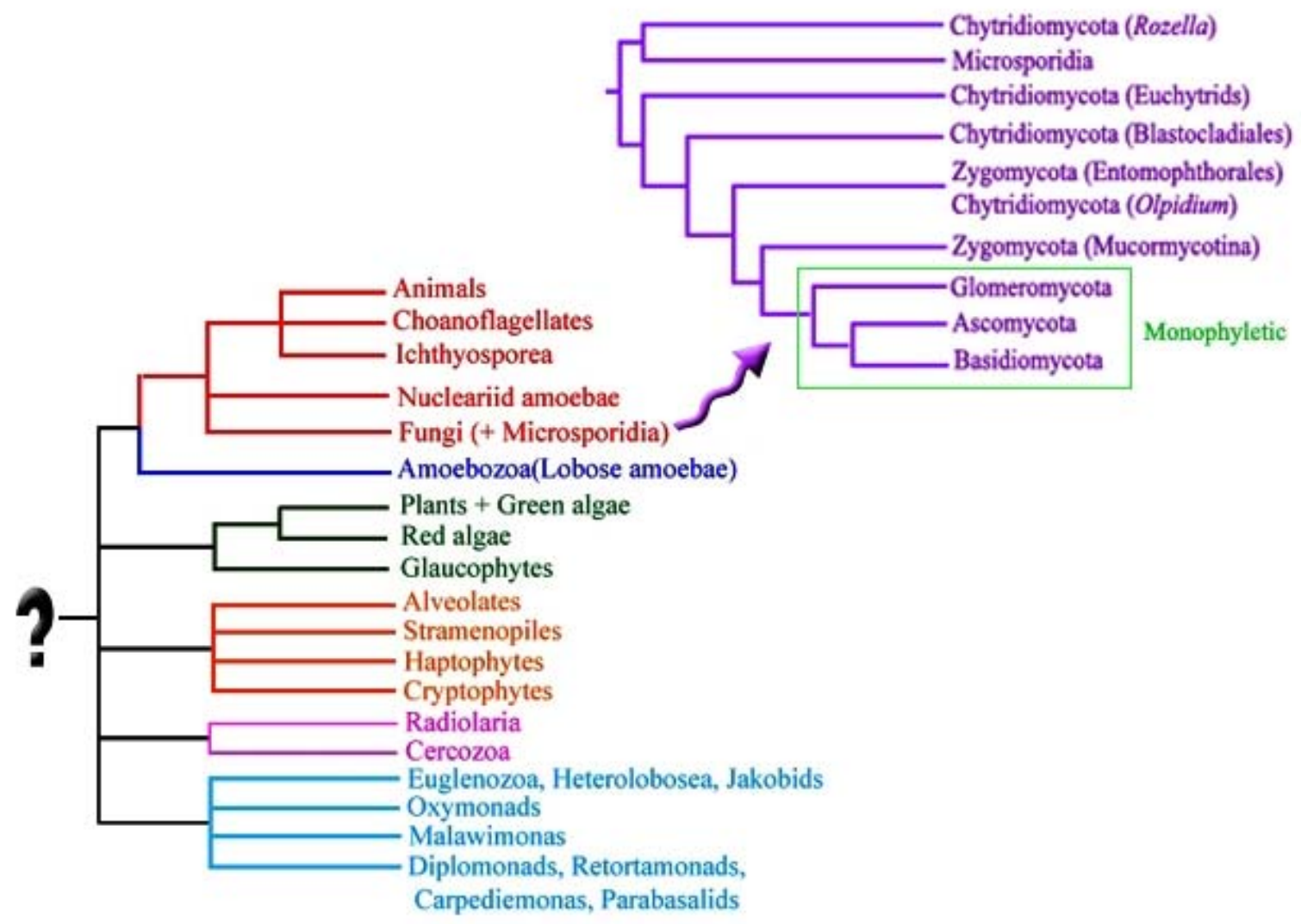

Figure 3 | Phylogenetic tree depicting the relationships of different groups of eukaryotes: Here the eukaryotes have been classified into six different groups which support the 'Six Super group classification' of eukaryotic phylogeny. Each colour represents one group. The Fungi has been placed closer to the animals compared to plants. Inset: The main branches of kingdom fungi. The Ascomycota and Basidiomycota are united as the dikarya. Their closest relatives seem to be the Glomeromycota that was previously included in the Zygomycota. Neither the Zygomycota nor the Chytridiomycota are monophyletic groups; instead they seem to be 'paraphyletic grades'. The microsporidia and Rozella branches seem to be basal to the all other fungi.

\section{Conclusion}

"Simple plants without chlorophyll"-is the statement appropriate for fungi? In the past decades biologist included them within the plant kingdom because of the presence of cell wall and production 
of spores. This classification has been changed in recent years, and according to many molecular phylogenics, they are the "sister group" of multicellular animals. Though they are still placed in plant kingdom, ample of recent researches regarding cell wall components, some specific amino acids and proteins, mitochondrial anatomy, cytochrome system, mode of nutrition, biochemical pathways and sequences of several proteins find much more similarities between fungi and animals than plants (FIG. 4). However, in spite of the large number

1. Porta, G. B. in Phytognomica, Orazio Salviani, Naples, Italy (1588).

2. Martin, G. W. Are Fungi Plants? Mycologia 47, 779791(1955).

3. Sachs, J. in Text Book of Botany, Oxford University Press, Oxford (1875).

4. Brefeld, $O$. in Untersuchungen aus dem Gesammtge-biete der Mykologie. VIII. Heft: Basidiomyceten III. Autobasidiomyceten, Leipzig, Germany: Arthur Felix, (1889).

5. Bessey, E. A. in A textbook of Mycology, Philadelphia: P. Blakiston's Son \& Co. Inc (1935).

6. Bessey, E. A. Some Problems in Fungus Phylogeny. Mycologia 34, 355-379 (1942). of evidences supporting the close relationship of fungi with the animals, few evidences have also been cited against this fungi-animalia clade of which the most important one is based on the work of Gouy. Gouy (1989) used transformed distance method and maximum parsimony method to compare the sequences of several kinds of macromolecules in organisms belonging to the 3 kingdoms: plants, animals and fungi. The analysis was based on the large subunit and small subunit rRNAs, 10 isoacceptor tRNA families and 6 highly

7. Fischer, A. Phycomycetes in Rabenhorst's Krypt-Fl. Deutschland, Oesterreich $u$ d. Schweiz. 1(4), 1-105 (1892).

8. Atkinson, G. F. Some problems in the evolution of the lower fungi. Ann. Mycol. 7, 441-472 (1909).

9. Borodin, J. Review of Chr. Gobi: Ueber die Gruppe der Amoeboideae [Original in Russian]. Bot. Centralblatt. 22, 35-38 (1885).

10. Cavers, F. The InterRelationships of Protista and Primitive Fungi. New Phytologist 14(2-3), 94-104 (1915).

11. Martin, G. W. Systematic Position of The Slime Molds and Its Bearing on The Classification of The Fungi. Bot. Gaz. 93, 421435 (1932).

12. Ramsbottom, J. Presidential Address: 'The expanding conserved proteins ${ }^{41}$. The results suggest that plants and animals are sibling kingdoms that have diverged more recently than the fungi.

Thus, extensive literature survey revealed that several different phylogenetic trees of Animalia, Plantae, and Fungi exist, differing in their views. But in the majority of cases, Fungi have been placed closer to Animalia than to Plantae (FIG. 3). In other words, animals have a more recent common ancestor with fungi than with plants.

knowledge of mycology since Linnaeus'. Proc. Linn. Soc. Lond. 151(4), 280-367 (1941).

13. Langeron, M. \& Vanbreuseghem, R. in Precis de Mycologie, ed. 2, Masson, Paris (1952).

14. Sussman, S. A. in The Fungal Organism, 2, Academic Press N.Y. 650653 (1996).

15. Ruiz-Herrera, J. in Fungal Cell Wall: Structure, Synthesis, and Assembly. CRC Press, Florida 91-120 (1992).

16. Carlile, M. J. \& Watkinson, $\mathrm{S}$. in The Fungi. Academic Press Ltd. San Diego, CA, (1994).

17. Cavalier-Smith, $\mathrm{T}$. in The Origin of the Fungi and Pseudofung. University Press, Cambridge, 340-376 (1987).

18. Boulter, D. \& Derbyshire, E. Cytochromes of Fungi. 
J. Exp. Bot. 8(2), 313-318 (1957).

19. Goodwin, T. W. \& Mercer, E. I. in Introduction to Plant Biochemistry. Pergamon Press, Oxford, 411 (1988).

20. Gaulin, E., Bottin, A. \& Duma, B. Sterol biosynthesis in oomycete pathogens. Plant Signal Behav. 5, 258-260 (2010).

21. Lejohn, H. B. Biochemical Parameters of Fungal Phylogenetics. in Evolutionary Biology Plenum Press, N.Y. and London 7, 79-120 (1974).

22. Shaw, R. The occurrence of $\gamma$-linolenic acid in fungi. Biochim. Biophys. Acta Lipids and Lipid Metabolism 98(2), 230-237 (1965).

23. Vogel, H. J. Two modes of lysine synthesis among lower fungi: evolutionary significance, Biochim. Biophys. Acta 41, 172-173 (1960).

24. Cavalier-Smith, T. in Systems-Eukaryote

kingdoms: Seven or Nine? University Press, Cambridge, 14, 461-481 (1981).

25. Baldauf, S. L. \& Palmer, J. D. Animals and fungi are each other's closest relatives: congruent evidence from multiple proteins. Proc. Natl. Acad. Sci. U S A. 90(24), 1155862 (1993).

26. Belfield, G. P. Translation Elongation Factor-3 (EF-3): An Evolution Eukaryotic Ribosomal Protein? J. Mol. Evol. 41, 376-387 (1995).

27. Keeling, P. J. et al. The tree of eukaryotes. Trends Ecol. Evol. 20, 670-676 (2005).

28. Borchiellini, C., et al. Phylogenetic analysis of the Hsp70 sequences reveals the monophyly of Metazoa and specific phylogenetic relationships between animals and fungi, Mol.
Biol. Evol. 15(6), 647-55 (1998).

29. Simpson, A. G., Inagaki, Y. \& Roger, A. J. Comprehensive Multigene Phylogenies of Excavate Protists Reveal the Evolutionary Positions of "Primitive" Eukaryotes. Mol. Biol. Evol. 23 (3), 615-625 (2006).

30. Chakraburtty, K. Translational regulation by ABC systems. Res. Microbiol. 152(3-4), 391-9 (2001).

31. Kuma, K. Phylogenetic position of Dictyostelium inferred from multiple protein data sets. J. Mol. Evol. 41, 238-246 (1995).

32. Lang, B. F. The mitochondrial genome of the fission yeast Schizosaccharomyces pombe: highly homologous introns are inserted at the same position of the otherwise less conserved cox 1 genes in Schizosaccharomyces pombe and Aspergillus nidulans. EMBO J. 3, 2129-2136 (1984).

33. Scazzacchio, C. The Natural History of Fungal Mitochondrial DNA. in Evolutionary Biology of the Fungus, Cambridge University Press, 53-73 (1987).

34. Inagaki, Y. et al. Directionally evolving genetic code: the UGA codon from stop to tryptophan in mitochondria. J. Mol. Evol. 47(4), 378-84 (1998).

35. Sogin, M. L. Early evolution and the origin of eukaryotes. Curr. Opin. Genet. Dev. 1, 457-463 (1991).

36. Sogin, M. L., Hinkle, G. \& Leipe, D. D. Universal tree of life. Nature 362, 795 (1993).

37. Sogin, M. L. \& Silberman, J. D. Evolution of the protists and protistan parasites from the perspective of molecular systematics. Int. J. Parasitol. 28, 11-20 (1998).

38. Adl, S. M., et al. The new higher level classification of eukaryotes with emphasis on the taxonomy of protests. J. Eukaryot. Microbiol. 52, 399-451 (2005).

39. Koonin, E. V. The Biological Big Bang model for the major transitions in evolution. Biol. Direct. 2, 21 (2007).

40. James, T. Y. et al., Reconstructing the early evolution of Fungi using a six-gene phylogeny. Nature 443, 818-822 (2006).

41. Gouy, M. \& Li, W. H. Molecular phylogeny of the kingdoms Animalia, Plantae and Fungi. Mol. Biol. Evol. 109-122 (1989). 